\title{
Theme: Pediatric Cardiology
}

\section{Q}

Coronary artery complication in Kawasaki disease and the importance of early intervention (JAMA Pediatr. 2016 Dec 1;170(12):1156-1163. doi: 10.1001/ jamapediatrics. 2016.2055).

The authors of this systematic review retrieved clinical studies that compared corticosteroids plus intravenous immunoglobulin (IVIG) therapy with IVIG therapy alone in treating patients with Kawasaki disease in the databases until July 2015. Studies either using corticosteroids as initial therapy or as rescue therapy were included. Sixteen comparative studies characterizing 2746 patients were analyzed. The rate of coronary artery abnormalities was significantly lower in adjunctive corticosteroids therapy than in IVIG therapy (OR $0.42 ; 95 \% \mathrm{CI}, 0.27,0.67)$. The overall efficacy was negatively correlated with the duration of illness before corticosteroid therapy $(P<0.001)$. Subgroup analysis, including studies using corticosteroids plus IVIG as initial therapy, showed a more advantageous effect than IVIG alone regarding coronary artery abnormality prevention (OR $0.32 ; 95 \%$ CI $0.18,0.56$ ), whereas this benefit was not found in a subgroup of studies using corticosteroids as rescue therapy. Further analysis showed that patients predicted at baseline to be at high risk of IVIG resistance seemed to obtain the greatest benefit from adjunctive corticosteroid therapy regarding coronary artery abnormality prevention (OR $0.24 ; 95 \%$ CI $0.12,0.47$ ). The fever duration was significantly reduced in the corticosteroids group. The favorable effects of corticosteroids were conferred without an increased risk of adverse events. The authors concluded that high-risk patients with Kawasaki disease benefit greatly from a timely and potent adjunctive corticosteroid therapy strategy.

Management of undernutrition and failure to thrive y in children with congenital heart disease in low- and middle-income countries (Cardiol Young. 2017 Dec; 27(S6):S22-S30. doi: 10.1017/S104795111700258X).

This review summarizes the existing evidence on prevalence of undernutrition and congenital heart disease (CHD) and its impact on surgical outcomes with a focus on implications for low- and middle-income countries. There are multifactorial causes for failure to thrive, including innate growth potential, severity of cardiac disease, increased energy requirements, decreased nutritional intake, malabsorption, and poor utilization of absorbed nutrition. The authors stressed the importance that failure of growth is not a contraindication to surgical repair, and patients should receive surgical repair where indicated as soon as possible. There is very little evidence to support the notion that lower weights are associated with poorer surgical outcomes. The article provides an approach to nutrition in the child with failure to grow or to gain weight and suggests an algorithm for nutritional management in the early postoperative period in low-resource environments. The review conclude that nutritional support requires specific attention and allocation of resources, including appropriately skilled personnel. Thereafter, it is essential to monitor growth and development and to identify causes for failure to catch-up or grow appropriately.

Educational achievement of children with congenital heart disease (Early Human Dev. 2018;128:27-34).

In view of improving survival rates of children with congenital heart disease (CHD), there is increasing interest in their quality of life. This cross-sectional study, using an online survey of patients registered in database of the German National Register for Congenital Heart Defects, assessed the educational achievement of patients with CHD. The large majority (83.4\%) of study participants $(n=2609)$ were enrolled at a conventional elementary school and started school at the age of 6 years or below (73.3\%). In total $45.7 \%$ of graduated study participants graduated with the qualification necessary to study at any university. In terms of analysis as per severity of CHD, $57.3 \%$, $47.5 \%$ and $35.1 \%$ of patients with mild-, moderate-, and severe CHD, respectively, attained a high school diploma. Authors concluded that the majority of participating CHD patients had a standard school career, suggesting that a normal school career is possible for all CHD patients. These results have important implications for Indian children with CHD where parents are often asked to get certificate of fitness from doctors for possibility of 'normal' schooling.

Survival and associated risk factors for mortality among infants with critical congenital heart disease in a developing country (Pediatr Cardiol. 2018;39:1389-96).

This retrospective cohort study from Malaysia examined Critical congenital heart disease (CCHD) survival and the risk factors for CCHD mortality. A total of 491 CCHD cases were included in the study, with an overall mortality rate of $34.8 \%$ $(95 \%$ CI 30.6, 39.2). The intervention/surgical mortality rate was $9.8 \%$ within 30 days and $11.5 \%$ in $>30$ days after surgery, and $17 \%$ died before surgery or intervention. The median (IQR) age at death was $2.7(1,7.3)$ months. The CCHD survival rate was $90.4 \%(95 \%$ CI $89 \%, 91.8 \%)$ at 1 month, $69.3 \%$ (95\% CI $67.2 \%, 71.4 \%)$ at 1 year, $63.4 \%(95 \%$ CI $61.1 \%, 65.7 \%)$ at 5 years, and $61.4 \%(95 \%$ CI $58.9 \%, 63.9 \%)$ at 10 years. Weight $<2 \mathrm{~kg}$ at diagnosis, associated syndromes, poor pre-operative condition, and non-duct-dependent CCHD were independent risk factors for poor survival. The authors concluded that the mortality and survival of infants with CHD vary with the severity of the lesion, with CCHD having high mortality and low short- and medium-term survival rates.

Balaganesh KarmegaraJ ANd R Krishna KumaR* "rkrishnakumar@aims.amrita.edu 